\title{
Six-Hertz Spike and Wave Epilepsy
}

\author{
Fermina Pirmohamed ${ }^{1 *}$, Soundarya Gowda ${ }^{2}$ \\ ${ }^{1}$ Clinical Neurophysiology Fellow, Virginia Commonwealth University, Department of Neurology, Richmond VA, USA \\ ${ }^{2}$ Assistant Professor of Neurology, Virginia Commonwealth University, Department of Neurology, Richmond VA, USA
}

Received: February 10, 2016; Accepted: March 17, 2016; Published: April 11, 2016

*Corresponding author: Fermina Pirmohamed, Clinical Neurophysiology Fellow, Virginia Commonwealth University, Department of Neurology, Richmond VA, 23298, USA, Tel: 804-828-9583; Fax: 804-828-6373; Email: fermina.pirmohamed@vcuhealth.org

\begin{abstract}
Introduction: A six-hertz spike and wave pattern has been reliably described as a benign rhythm of reactivity and drowsiness and occurs in $2.5 \%$ of adolescents and adults. Seizures are more commonly derived from a higher frequency and larger amplitude pattern on electroencephalography. This is a case of a six-hertz spike and wave pattern associated withgeneralized tonic-clonic seizures from a possible focus of cortical dysplasia.
\end{abstract}

Case Description: A nineteen-year-old male presented to the hospital after his second witnessed seizure. Seizure semiology is generalized tonic-clonic in nature and unprovoked. His neurologic examination was non-focal. His general examination and basic labs including an infectious work-up and full drug screen were negative. He was admitted for observation and found to have a sixhertz generalized spike wave pattern on his electroencephalogram. A contrasted MRI of the brain was performed and revealed a right frontal heterogeneous lesion adjacent to the anterior horn of the lateral ventricle. He was subsequently placed on an anti-epileptic medication and reports being seizure free at six-month follow-up.

Discussion: This case illustrates a patient where a six-hertz spike wave pattern is correlated with seizures and cortical dysplasia. Historically, a six-hertz spike wave pattern has been coined "phantom spike and wave" as it has been correlated with reactivity and known to be a rhythm of unknown significance. However, in the correct clinical circumstance, further investigation of this electroencephalographic pattern may be warranted in order to ensure appropriate therapy and management.

In this report we discuss a case of epileptic seizures associated with a benign six-hertz spike and wave electroencephalographic pattern originating from focal cortical dysplasia. A phantom six-hertz spike and wave pattern has not been reported in literature in relation to cortical dysplasia and epilepsy.

Keywords: Electroencephalogram (EEG); Neurophysiology; Cortical dysplasia; Epilepsy; 6-Hz spike wave; Seizure

\section{Introduction}

In this case report we would like to present a pattern of six-Hertz spike and wave electroencephalographic pattern associated with focal cortical dysplasia and heterotopia.

\section{Case Report}

A nineteen-year-old male presented to the hospital after having his second witnessed seizure. His seizure was described as arising out of wakefulness following a night of sleep deprivation. He was witnessed to have generalized tonic clonic activity at the onset without focality, with foaming at the mouth for approximately three to four minutes after which he had fifteen to twenty minutes of post-ictal fatigue without confusion. He denied tongue biting, bowel or bladder incontinence and any aura or prodrome. One year prior to this event, he had a similar episode but details regarding workup were not known, and he was not on any treatment. He has never had febrile seizures as a child according to his mother and there is no family history of epilepsy. He denied other potential provoking factors.

His neurologic examination was non-focal. His general examination and basic labs including an infectious work-up, electrolytes, liver function tests, urinalysis and full drug screen were also negative. A routine EEG showed six-hertz spike wave pattern as an interictal abnormality [Figure 1]. Specifically, it showed a symmetric and well-modulated background with brief, intermittent, frontally predominant six-hertz spike-wave discharges. The spikes were better formed with maximal surface negativity in the right frontal area. A contrasted MRI of the brain was performed revealing an area of heterotopia in the right frontal area adjacent to the anterior horn of the lateral ventricle with corresponding enlargement of the right temporal horn and tip with no evidence of hippocampal sclerosis or atrophy [Figure 1]. He was subsequently placed on an anti-epileptic medication and reports being seizure free on monotherapy of Keppra $1500 \mathrm{mg}$ twice a day at twelve-month follow-up.

\section{Discussion}

A six-hertz spike and wave pattern has been reliably described as a benign rhythm of reactivity and drowsiness that occurs in $2.5 \%$ of adolescents and adults. It occurs during relaxed wakefulness, drowsiness, stage I or light sleep and disappears during deeper stages of sleep. This pattern manifests in generalized bursts, predominantly symmetric and synchronous over the anterior or posterior regions of the head depending on gender. The morphology consists of sharp contoured positive 


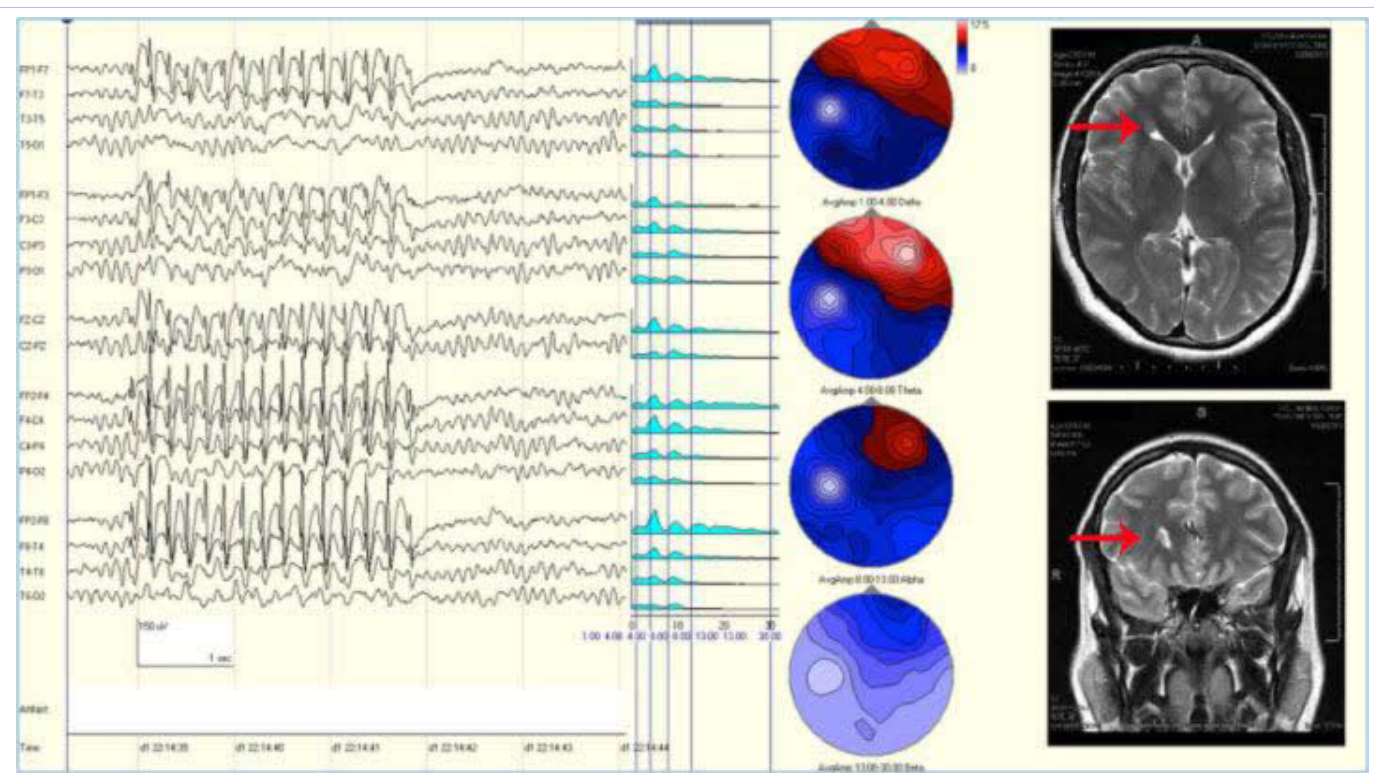

Figure 1: From left to right EEG shows frontally predominant 6-Hz spike and wave activity in a bipolar montage, a voltage map and voltage topography shows a dipole that has maximal negativity in the right frontal area and axial and coronal T2 MRI images of the brain shows a corresponding right peri-ventricular cortical dysplasia.

spikes alternating with rounded negative waves. They occur in short runs usually lasting less than 5 seconds and as the name would imply, the frequency is six hertz [1]. Demographically, it occurs more often in women and in the third or fourth decade of life [2]. Clinical correlates of this electroencephalographic activity are thought to be head injury and excessive drug use, although it has also been associated with psychiatric symptoms, brain tumors, cerebrovascular disorders, post-traumatic encephalopathies, cerebral degenerative diseases and other central nervous system diseases [3,4]. Six-hertz spike and wave activity was coined as "phantom" activity because it is thought to be a normal physiological cerebral discharge. This is confirmed by an extensive EEG survey of a large cohort of men in the US army [2]. Furthermore, a study shows six-hertz spike and wave bursts occurring during REM sleep, which would correspond to its physiologic occurrence [5]. The EEG pattern is generalized in nature and not derived from focal dysplasia or abnormality.

This case illustrates a unique presentation of a six-hertz spike and wave pattern occurring in concordance with generalized tonic-clonic seizures from a likely focus of cortical dysplasia. On EEG, the waveforms have a right frontal maximal surface negativity and are not truly generalized in distribution. These differences prompted further workup with neuroimaging and management was altered leading to better outcomes for this patient.

\section{References}

1. Ebersole, J, TA Pedley. Current practice of clinical electroencephalography. 3rd ed. Philadelphia: Lippincott Williams \& Wilkins; 2003.

2. Tharp B R. The 6-per-second spike and wave complex. The wave and spike phantom. Archives of neurology. 1966;15(5):533-537. doi:10.1001/archneur.1966.00470170087009.

3. Silverman D, Theda Sannit. Clinical correlates of the spike-wave complex. Electroencephalography and clinical neurophysiology. 1954;6:663-669. doi:10.1016/0013-4694(54)90093-0.

4. Hughes J R, R E Schlagenhauff, Margita Magoss. Electro-Clinical Correlations in the Six Per Second Spike and Wave Complex. Electroencephalography and clinical neurophysiology. 1965;18(1):7177. doi:10.1016/0013-4694(65)90148-3.

5. Gelisse P, A Crespel. Phantom spike-and-wave bursts during REMsleep. Neurophysiologie clin. 2008;38(4):249-253. doi: 10.1016/j. neucli.2008.04.002. 